Revista InTERnACIONAL de Sociología (RIS)

Procesos de exclusión social en un contexto de incertidumbre VOL. 72, EXTRA 1, 15-24, JUNIO 2014

ISSN: 0034-9712; elSSN: 1988-429X

DOI:10.3989/ris.2013.03.18

\title{
LOS RIESGOS DE EXCLUSIÓN SOCIAL EN UN CONTEXTO DE INCERTIDUMBRE
}

\author{
SOCIAL EXCLUSION RISKS IN AN \\ UNCERTAINTY CONTEXT
}

\author{
Robert Castel* \\ École des Hautes Études en Sciences Sociales. París. Francia.
}

\begin{abstract}
RESUMEN
En las siguientes líneas se realiza una disertación sobre la gran transformación de los procesos de exclusión social que, agravados por la crisis, tiene como resultado una multiplicación de las categorías sociales con dificultades de integración. Los procesos que les afectan tienen el mismo origen y se apoyan en una creciente degradación del mercado de trabajo y a la creciente asociación del trabajo y la pobreza, que conlleva la construcción de una frontera difusa entre éste y la asistencia, la pérdida del derecho de auxilio, a un proceso de culpabilización de las coberturas sociales, a un cambio de tendencia del welfare al "work far" y, en definitiva, a una pérdida de reconocimiento de los derechos, que requieren la colectivización de los medios para combatirlos y la movilización de los diferentes tipos de colaboradores concernidos en la defensa de los derechos de las categorías cada vez más numerosas.
\end{abstract}

\section{Palabras clave}

Crisis; Exclusión social; Pobreza; Trabajo.

\begin{abstract}
Below we realise a dissertation about the great transformation of social processes of social exclusion, which causes the multiplication of those social categories with difficulties to integration, aggravated by the current crisis situation. The processes that affect them have the same origin and are based on an increasing deterioration of the labour market and an increasing association of work and poverty, that entails the building of a diffused frontier between it and the assistance, the loss of the right to help, to a process of blaming of social coverage, to a tendency change from welfare to work far and, in short, a loss of the reconnaissance of rights, which need the collectivization of means to fight against them and the actions of the different types of contributors who are concerned about the protection of rights of more and more numerous categories.
\end{abstract}

\section{KEYWORDS}

Crisis; Poverty; Social Exclusion; Work.

"El siguiente texto es una adaptación de la conferencia inaugural dictada por el Dr. Robert Castel en el Aula Magna de la Universidad de Jaén el día 21 de febrero de 2011, con motivo del III International Congress on Intelectual Disability ( "Advancing in social responsability" Autonomy / Inclusion / Sensibilization). Desgraciadamente, el profesor Castel murió dos años después, el 12 de marzo de 2013.

Traducción: Patricia Jiménez Angulo. Revisión: Eva María Sotomayor Morales. 


\section{INTRODUCCIÓN}

Los problemas que plantea la exclusión ya no se encuentran únicamente en los márgenes de la sociedad. La amenaza de la exclusión también se cierne sobre las personas y los grupos que habían podido integrarse en ella, que parecían tener la capacidad de llevar una vida "digna", y que puede que, si no se convierten en excluidos, estén en todo caso condenados a llevar una vida precaria sumida en la incertidumbre de qué va a ser de ellos el día de mañana, e incapaces de controlar su destino.

Este cambio en la problemática general de la exclusión se observa por ejemplo en el colectivo de personas con discapacidad y en el propósito de nuestra sociedad de mejorar la calidad de vida de estas personas. Seguramente este ámbito plantea problemas específicos y exige un tratamiento especializado pero, al mismo tiempo, estos problemas surgen hoy en un nuevo contexto en el cual, la discapacidad ya no constituye una excepción. A partir de ahora hay similitudes entre los problemas que plantean estas personas y los que plantean los trabajadores precarios o los grupos de individuos vulnerables que se enfrentan a formas de hábitat degradadas o a factores de disociación familiar con el desarrollo de las familias monoparentales. Hay toda una zona de expansión de nuestras sociedades, marcada por formas de vida degradadas en las que los individuos viven, en cierto sentido, al día, tal y como se procedía hace ya algunos años.

Lo que voy a abordar es la expresión de una dinámica profunda que supera las fronteras de lo que llamamos "la globalización", cuyos efectos se sufren tanto en España como en Francia, probablemente con ciertas diferencias sobre las cuales podríamos debatir en otro contexto. En este sentido se evidencia que ha habido una transformación bastante profunda de ámbito de la exclusión que es necesario tener en cuenta y que, en primer lugar, es oportuno aportar una explicación de este proceso.

Concretamente en este punto puedo expresarme con relativa precisión, ya que estuve implicado en prácticas de lucha contra la exclusión antes de que se produjera un cambio de tendencia. A finales de los años 60 y durante los años 70, trabajé durante bastante tiempo sobre la psiquiatría y la medicina mental. Pero lo hice no solo de forma académica, también participé activamente en lo que en aquel momento bautizamos de manera aproximativa como la "anti-psiquiatría". En particular habíamos fundado entre unos cuantos un gran grupo llamado "La Red interna de alternativa a la psiquiatría", con algunos colegas italianos, en concreto con Franco Basaglion, el gran psiquiatra italiano del cual algunos de ustedes habrán oído hablar, y cuya acción culminó con el voto de la ley 180 en Italia, que abolió los hospitales psiquiátricos en dicho país. Nuestro objetivo era el de luchar contra la segregación social de los enfermos mentales cuyo encerramiento en los hospitales psiquiátricos, a menudo de por vida, constituía la manifestación más escandalosa de la vida social. Me acuerdo incluso de haber viajado a Barcelona en 1973, estando España todavía bajo el régimen franquista, a causa de que un psiquiatra español, Ramón García, fue procesado por tratar de abrir el hospital psiquiátrico de Barcelona y "desinstitucionalizar" a los enfermos mentales como pretendíamos por aquel entonces. Por este motivo algunos 
de nosotros fuimos para apoyarle de forma un tanto clandestina en el contexto español de la época.

En este sentido, en los años 70, grupos concretos como los enfermos mentales, los presidiarios, los minusválidos y algunos "inadaptados" vivían apartados de la sociedad, fuera del régimen común de intercambios sociales y consentido por aquellos a quienes les estaba permitido hablar de manera totalmente legítima de exclusión social, existiendo una clara diferencia entre la manera en la que eran tratados y la vida social ordinaria. A algunos de nosotros esto nos parecía escandaloso puesto que, y es un punto sobre el que quiero insistir, nos encontrábamos en un periodo de desarrollo económico y de progreso social extraordinario.

Durante la década de los 70 , los principales países de Europa occidental y también España -aunque con cierto retraso debido a su situación particular-, vivían el final de un periodo, aun no siendo conscientes de ello. En estos países se podía constatar un progreso acompañado de un crecimiento económico y de derechos sociales, lo que entrañaba el comienzo de una visión optimista del futuro: "un mañana con más y mejor". Debido a ello, me parecía especialmente indigno que, mientras casi toda la población celebraba el progreso, había gente, como por ejemplo los enfermos de los hospitales psiquiátricos, a los que se les trataba de una manera que recordaba más a la Edad Media que a la sociedad civilizada de la que nos vanagloriábamos por el mero hecho de pertenecer a ella.

Para simplificar, podríamos decir que en estas sociedades que parecían dejarse llevar por una dinámica de progreso, estos grupos reducidos de los llamados "excluidos" aparecían más como islotes arcaicos, como una herencia obsoleta del pasado en vías de desaparición. Se trataba de un situación completamente diferente a la de hoy, porque estos problemas relacionados con la exclusión, en cierto sentido, se han extendido y afectan a nuevas categorías de población, lo que nos podría llevar a preguntarnos acerca de la pertinencia de continuar hablando con propiedad de la exclusión. En este sentido, tengo ciertas reticencias con respecto al uso demasiado generalizado del término exclusión, ya que, estrictamente hablando, estar excluido significa estar totalmente aislado de la sociedad, estar fuera de la sociedad. Sin embargo nadie se encuentra aislado de la sociedad propiamente hablando, salvo quizás en algunas situaciones límite como la de los "sin techo"; aunque incluso este colectivo, en ocasiones tiene buena relación, por ejemplo, con los agentes sociales.

El problema masivo es más bien el de la precariedad, el de la multiplicación de individuos o de grupos vulnerables que se ven debilitados, que carecen de los recursos suficientes para garantizar su independencia económica y social y que en última instancia, pueden caer en lo que llamamos la exclusión.

Sin embargo, en mi opinión, es necesario analizar estas situaciones en términos de proceso o de recorrido y no conformarse con emplear el término de exclusión. Por ejemplo hay personas que trabajan, tienen un jefe y un sueldo, pero bajo condiciones tan precarias y tan degradadas que en seis meses pueden verse totalmente desprovistos de su nivel de vida. Incluso alguien que ocupe una vivienda cuyo alquiler consigue pagar 
a duras penas, puede verse desprovisto de vivienda al tener un contrato laboral de seis meses de duración. Es a esta nebulosa de situaciones inestables y frágiles, a la que hay que referirse para posicionarse con respecto a la exclusión de la sociedad actual.

Aunque no de forma detallada, me gustaría trazar las directrices de esta dinámica nueva que se despliega desde hace algo más de treinta años en el marco de lo que comúnmente llamamos "La Crisis", pero de la cual no hemos comprendido en un primer momento la importancia ni la gravedad. A su inicio se pensó que se trataba de un estancamiento provisional de esta dinámica de desarrollo que se desplegaba desde la Segunda Guerra Mundial; se esperaba "la recuperación" como se decía en aquella época, pero de hecho ha hecho falta comprender, aunque hayamos necesitado un tiempo para hacerlo, que la situación tenía un mayor gravedad y que nos hundíamos en esta crisis en la que aún nos encontramos, como nos lo ha mostrado el terrible cataclismo del otoño de 2008.

Hemos salido del capitalismo industrial y de las formas de regularización que se consiguieron en dicha etapa y hemos entrado en un nuevo régimen de capitalismo más salvaje que juega a la competencia exacerbada a nivel planetario ("globalización") bajo la hegemonía cada vez más brutal del capital financiero e instaurando la especulación por la especulación.

En este sentido, hemos de cuestionarnos lo que llamamos "el compromiso social del capitalismo industrial", teniendo en cuenta la multiplicación del número de individuos que se ven apartados hacia los márgenes de la sociedad, abandonados a la precariedad, viviendo en la incertidumbre del mañana dentro de una inseguridad social creciente.

Es sabido que el capitalismo industrial en Europa comenzó a implantarse de una manera muy salvaje. Sólo hay que ver las condiciones de los proletarios del siglo XIX, cuando eran despreciados y se les trataba miserablemente, perdiendo literalmente la vida mientras se la ganaban en una inseguridad total. Sin embargo tras una larga historia de más de un siglo, debido a las luchas y a los conflictos a veces violentos, se alcanzó una cierta forma de equilibrio o de compromiso social entre "los vulnerables del mercado del capital", dicho en términos de rentabilidad y de competitividad de las empresas, que desembocó en este gran crecimiento, por una parte y, por otra, en la emergencia del asalariado en términos del mundo laboral, que se mantenía en una posición subordinada por estar sometido al régimen capitalista.

La Revolución no tuvo lugar, al menos en Europa occidental, pero a cambio los trabajadores se beneficiaban de una seguridad y de unas protecciones, así como de un estatus laboral sólido. Esta estructura es la que llamamos "la sociedad salarial", término que no expresa la situación de que la gran mayoría de la población sea asalariada, aunque este fuera el caso. En el caso de Francia, hay en la actualidad un $90 \%$ de la población de asalariados dentro de la población excluida. Pero esto significa sobre todo que la gran mayoría de la población obtiene derechos principalmente de su trabajo, para el trabajador y su familia. En este sentido, sus "derechohabientes" son el fruto de la instauración de una seguridad social generalizada que sobre la base de un trabajo estable, cubre a la gran mayoría de la población contra los principales riesgos sociales. 
Y esto es precisamente lo que se está desmoronando con "la crisis" y con el empuje del nuevo régimen capitalista.

Desde los años 70 observamos dos manifestaciones principales de la degradación del trabajo, como son la instalación de un paro masivo y la precarización creciente de las relaciones laborales. En cuanto al desempleo masivo, hay que decir que este alcanza en Francia, en Europa occidental, pero también en Estados Unidos, una media del 10\% (en España afecta incluso al $20 \%$ de la población), pero también hay una precarización creciente de las relaciones laborales que se sitúan por debajo del estatus del trabajo que dominaba en la época precedente, tal y como, lo he caracterizado antes debido a varias cuestiones: (i) su duración estaba relativamente garantizada (principalmente los contratos de duración determinada), (ii) estaba bien protegido por el derecho laboral, (iii) con un salario que, supuestamente, era más o menos decente (al menos el Salario mínimo interprofesional creciente francés o Salario mínimo interprofesional español), (iv) el cual lleva incorporadas importantes prestaciones sociales (seguros contra accidentes, prestación por desempleo, derecho a la jubilación).

En torno al $60 \%$ de los trabajos son estables o lo parecen, al menos en términos de stock o de fondo; ahora bien, en términos de flujo o de entradas en el mercado de trabajo, hay sobrados motivos de alarma puesto que alrededor del $73 \%$ de estas entradas, generalmente protagonizadas por jóvenes, dependen de formas de empleo tildadas de atípicas, como el contrato de duración determinada, de carácter temporal y a tiempo parcial. Ello es la nueva tendencia, y si este proceso se prolonga sin fin, dejará de tener sentido que tildemos de "atípicas" a estas formas de trabajo que pueden convertirse en las predominantes. Además, estas formas están lejos de ser las únicas que se desarrollan en condiciones inferiores a las del asalariado clásico. De hecho, en el marco de la política de lucha contra el paro de corta duración, están los llamados "contratos subvencionados", que se prolongan con opción a ser prorrogados y que afectan generalmente a jóvenes que están buscando un empleo. También existe un gran elenco de prácticas que a menudo no son remuneradas y que rara vez conducen a un trabajo real: existen contratos cortos no prorrogables que se dan incluso en las administraciones y los servicios públicos, ámbitos que son considerados como bastiones de trabajo estable, además del desarrollo de servicios voluntarios a personas.

El hecho es que hay varios miles de personas que trabajan, pero lo hacen en condiciones claramente inferiores al estatus del trabajo, por lo que podríamos decir que en la actualidad tendríamos que empezar a replantearnos lo que hay que entender por precariedad. Durante mucho tiempo hemos pensado que se trataba de una situación provisional un tanto penosa, pero que no tenía nada de dramático. En el caso de los jóvenes concretamente, el hecho de limitar relativamente el comienzo de una trayectoria profesional ocupando algunas actividades provisionales, podía incluso enriquecer la experiencia profesional del individuo antes de instalarse en un "trabajo a largo plazo". No obstante, esta situación podría convertirse en la norma y podríamos instalarnos en la precariedad, ante la posibilidad de que esta pueda transformarse en un estado. Quiero destacar que he propuesto hablar de precariedad para designar esta condición, que 
puede adquirir un carácter permanente 0 al menos prolongarse a largo plazo. En relación a ello, hay un tipo de estrato de la división del trabajo que se instalaría por debajo del asalariado, si por asalariado entendemos una condición social fundada sobre el estatus del empleo tal y como lo he caracterizado.

Esto podría significar una transformación de la condición de los trabajadores tan grave como la existencia de un paro masivo, puesto que se traduce en una alteración profunda del estatus del trabajo. La reaparición desde hace una década de la categoría de "trabajador pobre" es una manifestación concreta de este hecho, no porque sea algo novedoso, sino todo lo contrario, ya que a través de la historia, la condición de los trabajadores ha estado casi siempre asociada a la pobreza. Sin embargo esta situación se consideraba arrinconada, y de hecho lo estaba, ya que se había superado con creces en la sociedad salarial con la hegemonía del estatus del trabajo. Hoy parece que más bien se está expandiendo, es decir, que cada vez hay más personas que trabajan y que mediante su trabajo no consiguen cubrir sus necesidades ni las de su familia, en caso de que la tengan, lo cual puede derivarnos en última instancia a consecuencias bastante paradójicas.

Por ejemplo, actualmente hay medidas que se presentan como una gran actuación social de los gobiernos. Una de ellas consiste en que las personas que se encuentran en paro y que se benefician de una prestación asistencial' se ven casi obligadas a aceptar un trabajo, cualquiera que sea, ya que podrán añadir a su prestación (renta mínima de inserción) la retribución de su trabajo, aunque este sea mediocre y esté mal pagado. Estas iniciativas nacen de una buena intención puesto que no es del agrado de ninguna persona ser "un asistido puro y duro"; sin embargo, se prevé que muchas personas van a continuar en esta situación y no se constituye como una etapa que conduzca a un empleo estable. De esta forma tendrán un salario, un jefe, serán trabajadores en cierta medida, pero al mismo tiempo y por el hecho de que este salario es claramente insuficiente y a todas luces precario, serán a su vez personas beneficiarias de asistencia social que reciben su prestación. Debido a ello, en la actualidad se construye una frontera difusa entre los ámbitos del trabajo y asistencia.

Al final de este razonamiento se podría formular una hipótesis, que temo que sea algo más que eso, y que aunque no sea muy tranquilizadora, merece una reflexión, ya que se podría estar constituyendo una sociedad de pleno empleo que no llegaría a serlo tal y como estaba caracterizada en el pasado debido principalmente a la hegemonía del estatus del trabajo. De hecho, este nuevo régimen de capitalismo parece incapaz de garantizar el pleno empleo, cuya prueba más palpable es el paro masivo.

Pero podría suceder que a dicho régimen no le interesara el pleno empleo e incluso que no lo quisiera, porque a sus ojos el empleo es costoso y puede lastrar la competitividad de las empresas. El estatus del empleo otorga a los trabajadores principalmente garantías y derechos que se conciben como trabas para el libre desarrollo de

${ }^{1} R S A$ o renta de solidaridad activa en Francia, o Renta Activa de Inserción en España. 
las empresas y del mercado. En cambio este capitalismo requiere sagacidad e incluso, si es posible, una ocupación total. Este es además el lema de la OCDE, ya que el trabajo es necesario para crear riqueza. Entonces lo ideal es que todo el mundo trabaje pero bajo las condiciones más precarias y desprotegidas posibles. Así se podría entender esta presión que se ejerce hoy para que todo el mundo trabaje aunque sea bajo condiciones totalmente degradadas. Esto ya no es empleo, pero es la actividad en la cual la utilidad económica del trabajo se disocia al máximo de las garantías sociales que le fueron asignadas con el estatus del empleo.

En este caso, hay fuertes presiones a día de hoy que van dirigidas en esta dirección. Los estados nos repiten sin distinción que hay que trabajar a toda costa porque si no, se es un miserable asistido que vive a expensas de los que trabajan (de los que madrugan para levantar el país). Incluso se insinúa que los parados podrían serlo "de por vida", que es la manera eufemística de decir que son unos "gandules" y que tendrían que aceptar cualquier trabajo, ya que si todo el mundo tiene que trabajar a toda costa, esto implica que no hay que ser demasiado "escrupuloso" con el trabajo, e incluso no serlo en absoluto en términos de condiciones laborales, retribución laboral y protecciones asociadas al trabajo. Así es como nos convertimos en "trabajadores pobres", algo que no resulta nada agradable, pero que según esta ideología es incomparablemente mejor que ser un "parásito asistido". Y no solo hay trabajadores pobres propiamente hablando, existe todo este continente de precariedad en el que en última instancia podríamos reabsorber el desempleo, al menos en gran medida. Podría haber cada vez menos parados si hubiera cada vez más actividades de este tipo que se desarrollan en un estatus inferior al del empleo.

Esta dinámica de precarización se ha convertido en una dimensión básica de la coyuntura actual. La precariedad no tiende a ser un ámbito de la vida social que se sitúe únicamente en los márgenes o en la periferia de la sociedad, sino que atraviesa amplios sectores y desestabiliza la existencia de un número cada vez mayor de agentes sociales. Me parece que habría que reubicar la problemática dentro del conjunto de un colectivo, como son las personas con discapacidad intelectual porque tienen dificultades y no están bien integrados en la sociedad, no disfrutan de la totalidad de los derechos de la ciudadanía. Pero su situación no es verdaderamente excepcional, hay otros grupos sociales que están en una situación similar. No me refiero a ello al objeto de banalizar la discapacidad. Sin embargo podría ser un medio para ampliar las formas de comprensión en el marco de una lucha por los derechos que corresponden a las personas discapacitadas; también a distintas categorías de personas sometidas a la precariedad y al riesgo de exclusión. Porque hay otro punto que me gustaría resaltar a propósito de las transformaciones que se han sucedido en el marco de "la crisis".

Acabo de destacar la degradación de las condiciones de los trabajadores con el aumento del desempleo masivo y de la precariedad, pero en paralelo, también ha habido transformaciones importantes en las políticas y las intervenciones sociales que se han aplicado para hacer frente a estos cambios y que también tienen un impacto directo en las prácticas profesionales. Pienso en el desarrollo, una vez más, de las políticas públicas impulsadas concretamente en Europa desde 1980 en el marco de la OCDE con la 
activación como objetivo. Políticas que parten de una crítica de inspiración liberal de las intervenciones sociales que serían demasiado burocráticas, demasiado automáticas y que superarían sus prestaciones y sus servicios de forma incondicional. Lo que supondría descargar a los beneficiarios de la responsabilidad para instalarlos en la cultura de la asistencia creando consumidores pasivos de servicios.

En lugar de estos derechos incondicionales, habría que proceder a través de intervenciones específicas que susciten la participación de los beneficiarios, que los movilicen reforzando la capacidad de los individuos.

Esta es la gran consigna y creo que el hecho de "sustituir los gastos pasivos por los gastos activos" tiene que surtir efecto, concretamente en su capacidad de trabajo, en el sentido de incitarles u obligarles a aceptar o a retomar un trabajo: "making work pay" como decimos en inglés. Hay también un cambio de tendencia del welfare al work far , es decir, de "dispensar bienestar" a "obligar a trabajar", tendencia que se ha instalado en Estados Unidos con dureza. Pero esta perspectiva también ha inspirado, y mucho, la tercera vía en Inglaterra o las reformas recientes de la protección social en Alemania, que concretamente han endurecido las condiciones de subsidio y de desempleo. Lo que antes comenté acerca de la Revolución, es un ejemplo de esta política en Francia y supongo que habrá más orientaciones que vayan en la misma dirección. Las orientaciones de las políticas públicas plantean un problema difícil. Presentan características positivas ya que, es cierto que en una sociedad que cada vez es más móvil y está más fragmentada e individualizada, las intervenciones públicas ya no pueden proceder únicamente a través de grandes regulaciones colectivas de carácter general que aseguren protecciones homogéneas a todos. Es necesaria una cierta precisión en las intervenciones de una sociedad que se transforma cada vez más en una "sociedad de individuos" y en la que las trayectorias sociales están cada vez más diferenciadas. La idea de invertir en las personas para reforzar su capital, tal y como se dice, es totalmente defendible. Pero el riesgo radica en sustituir una lógica de comercialización por una lógica de protección que sea simplemente un intercambio, es decir, "yo te concedo una prestación o un servicio, pero tú, ¿qué me entregas a cambio?". No hay prestación sin contrapartida, este es el problema que se ve todos los días en el ámbito de las personas que requieren prestaciones sociales. Se pide mucho, a menudo demasiado, y a personas con dificultades que disponen de pocos recursos, y si no pueden responder a estas exigencias, se les culpabiliza. Es lo que los americanos llaman "culpar a la victima" y así es como se llega a "acusar" a los parados de ser prácticamente "parados voluntarios" o a los asistidos de ser parásitos que se conforman con la dependencia. Por eso me parece que la referencia al "derecho a" tiene que continuar siendo algo básico para los agentes sociales que trabajan con personas con dificultades, ya sean discapacitados, parados o jóvenes. Y es bajo esta condición por la que se les puede tratar como ciudadanos, no solo como personas desgraciadas que necesitan ayuda, también son personas de derecho que tienen derechos.

Me parece que la noción del derecho al auxilio, que además apareció en el momento de la Revolución francesa: "la nación debe prestar auxilio a sus ciudadanos desgracia- 
dos", esta noción del derecho al auxilio, es una gran idea que ha permitido dar paso a la asistencia privada y a la caridad facultativa. Hay una obligación de ayudar a los ciudadanos desgraciados y de esta forma no nos adentramos en una lógica comercial del intercambio sino en una lógica de servicios públicos con usuarios que tienen derechos, y así los individuos que dependen de otros pueden acceder a la autonomía para convertirse en ciudadanos iguales que los demás.

En mi opinión, en el marco de esta "crisis" a la que hemos hecho alusión antes, destacamos cada vez más la competitividad, la rentabilidad, las exigencias de la competencia y la búsqueda del resultado. Hay que reafirmar la importancia de esta referencia al derecho que creo que constituye el fundamento de las profesiones del sector social y la base de un servicio público de protección social.

Creo que las cuestiones prácticas no están disociadas de las dinámicas sociales, y es por lo que he resaltado esta "gran transformación" acaecida en el marco de la crisis que apareció hace ya algún tiempo. Como he comentado anteriormente, antes de esta crisis, en los años 60 y 70, aparentemente nos encontrábamos en una situación de mayor comodidad o menos complicada porque nos dejábamos llevar por una ola de progreso económico y social. Se trataba de reintegrar en la comunidad a un número limitado de excluidos y la comunidad podía ser más hospitalaria porque disponía de más recursos. Nos encontrábamos en una coyuntura de casi pleno empleo, lo que facilitaba la reinserción profesional de personas que tienen dificultades con el trabajo.

Hoy en día la coyuntura social es más dura, el empleo escasea, reina la competencia exacerbada entre los candidatos a un puesto de trabajo, la amenaza del paro y del despido está siempre presente, lo que en suma da lugar a una multiplicación de las categorías sociales que sufren dificultades de integración y se ven amenazadas por la exclusión, o al menos, están condenadas a formas de vida precarias que no les permiten llevar una vida autónoma.

La situación parece también ser más complicada que antes de "la crisis", pero quizás también podríamos decir que nos ofrece nuevas oportunidades, porque los problemas de todos aquellos que trabajen con el colectivo de personas con discapacidad intelectual, en el fondo son los mismos que los problemas que se plantean a la hora de luchar contra la degradación del trabajo, la precarización de los empleos; lo que nos ofrece la oportunidad de aliarnos con otros colaboradores, concretamente disponer de sindicatos de trabajadores que también se enfrentan al reto de combatir la precariedad.

Es por lo que, si los riesgos de exclusión se generalizan, quizás podríamos contemplar una colectivización de los medios para combatirla que movilizara a los diferentes tipos de colaboradores concernidos en la defensa de los derechos de las categorías de individuos, cada vez más numerosas, que no pueden acceder a la autonomía y cuyas deficiencias intelectuales son un caso más entre otros. Puede que termine con un apunte con matices de utopía , pero ¿por qué no?. Es cierto que estamos en un contexto de incertidumbre y no se puede decir que será del día de mañana, excepto si nos considerásemos profetas. Pero si el futuro es incierto, significa que lo peor tampoco es seguro, y que dependerá también, al menos en parte, de lo que hagamos o no hagamos 
en el presente para orientar su trayectoria. Por eso queda trabajo por hacer en el sentido de luchar por el pleno reconocimiento de los derechos de las personas en situación de vulnerabilidad social.

Robert CASTel (1933-2013) Sociólogo francés. Doctor en Letras y Ciencias Humanas. Fue profesor asistente de filosofía en la Facultad de Letras de la Universidad de Lille hasta 1967. Después del 68 enseñó sociología en la Universidad de Vincennes, que más tarde pasará a ser la Universidad de París VIII Vincennes-Saint-Denis. Fundador del Grupo de Análisis de lo Social y la Sociabilidad (GRASS). Asimismo, fue Director de estudios de la École des Hautes Études en Sciences Sociales de París. Sus obras analizan la constitución histórica de la sociedad salarial y su posterior disgregación desde principios de los años 70 (Las metamorfosis de la cuestión social, 1995), así como las consecuencias de ésta última para los individuos y las relaciones sociales: la exclusión social (lo que él llama la "desafiliación"), la vulnerabilidad y la fragilización crecientes.

RECIBIDO: 18/03/2013

ACEPTADO: 22/06/2013 